\title{
The expression of Claudin 1 correlates with $\beta$-catenin and is a prognostic factor of poor outcome in gastric cancer
}

\author{
JIE HUANG $^{1 *}$, JIANFANG LI $^{1 *}$, YING QU $^{2}$, JIANIAN ZHANG $^{1}$, LI ZHANG $^{1}$, \\ XUEHUA CHEN $^{2}$, BINGYA LIU ${ }^{1}$ and ZHENGGANG ZHU ${ }^{1}$ \\ ${ }^{1}$ Department of Surgery, Shanghai Institute of Digestive Surgery, Ruijin Hospital, Shanghai Jiao Tong University \\ School of Medicine, Shanghai 200025, P.R. China; ${ }^{2}$ Department of Surgery, Department of Obstetrics \\ and Gynecology, Women's Cancer Institute, Samuel Oschin Comprehensive Cancer Institute, \\ Cedars-Sinai Medical Center, Los Angeles, CA 90048, USA
}

Received November 30, 2013; Accepted December 30, 2013

DOI: $10.3892 /$ ijo.2014.2298

\begin{abstract}
Claudin 1 is one of the tight junction proteins, which are critical in the maintenance of epithelial integrity. Aberrant regulation of CLDN1 and its correlation with $\beta$-catenin have been discovered in malignant tumors. The present study aimed to investigate the expression profile and clinical relevance of CLDN1 and $\beta$-catenin. The protein levels of CLDN1 and $\beta$-catenin were examined using immunohistochemical staining. The characteristics of expression profile and prognostic value were analyzed using Pearson's $\chi^{2}$ test and Kaplan-Meier analysis, respectively. $\beta$-catenin overexpression and knockdown were used to investigate its role in regulating CLDN1 expression. We showed that CLDN1 was overexpressed in intestinal-type, presence of lymph node metastasis, higher TNM stage in gastric cancer patients and correlated with decreased overall survival. The characteristics of CLDN1 expression were associated with that of $\beta$-catenin. CLDN1 and $\beta$-catenin showed similar prognostic value in intestinal-type gastric cancers. $\beta$-catenin knockdown and overexpression in cell models revealed a positive relation between CLDN1 and $\beta$-catenin. Our study demonstrated that CLDN1 is a biomarker for intestinal-type gastric cancer with shorter survival. The expression of CLDN1 was strongly associated with $\beta$-catenin in gastric cancer patients and a gastric cancer cell model.
\end{abstract}

\section{Introduction}

Gastric cancer is the fourth most common cancer worldwide and is the second most common cause of cancer-related deaths.

Correspondence to: Dr Zhenggang Zhu, Department of Surgery, Shanghai Institute of Digestive Surgery, Ruijin Hospital, Shanghai Jiao Tong University School of Medicine, Shanghai 200025, P.R. China E-mail:zzg1954@hotmail.com

*Contributed equally

Key words: claudin 1, $\beta$-catenin, gastric cancer
Gastric cancer has a poor prognosis (1). Although the 5-year survival rate in patients with early-stage disease is $\sim 90 \%$, since the vast majority present with distant metastasis, the overall 5-year survival rate is typically $<20 \%$ (2). The 5-year survival rate has also been significantly correlated with the degree of tumor invasion, the presence of lymph node and/ or distant metastases and the TNM stage $(3,4)$. However, very limited number of molecules that have clinicopathological significance in gastric cancer has been discovered.

Claudin 1 (CLDN1) is one of the integral membrane proteins that constitute tight junctions. Tight junctions are essential for the tight sealing of cellular sheets and maintaining homeostasis (5). Absence of tight junctions or defective tight junctions is associated with the development of the neoplastic phenotype in epithelial cells (6). Thus it is accepted that the disruption of tight junctions leads to loss of cohesion, invasiveness and the lack of differentiation, thereby promoting tumorigenesis. CLDN1 is found to regulate intestinal epithelial homeostasis through the modulation of Notch-signaling (7). Many chemicals and nutrition can regulate CLDN1 expression through different pathways $(8,9)$ to maintain the integrity of intestinal barrier function.

The complexity of CLDN1 was proposed because its dual role as a tumor suppressor and promoter, as well as a positive and negative prognostic factor in different cancers, including breast $(10,11)$, gastric (12), ovarian (13), lung (14) and colon (15-17). Decreased expression of CLDN1 is correlated with recurrence status in breast cancer (18). Similarly, studies on lung cancer also revealed CLDN1 expression is correlated with better survival $(14,19)$. Opposite findings suggest that CLDN1 is a negative prognostic factor. Alteration of CLDN expression may affect permeability at tight junction, possibly increasing the diffusion of nutrients and other extracellular growth factors to promote cancer cell growth, survival and motility in gastric cancer (20). Claudin 1 induces epithelialmesenchymal transition through activation of the c-Abl-ERK signaling pathway (21).

CLDN1 is involved in many cellular functions including cell proliferation, survival and metastasis. CLDN1 knockdown in breast cancer cells significantly decreased cell migration and the expression EMT markers (11). CLDN1 expression 
was correlated to anoikis resistance in colon cancer (22). CLDN1 upregulates the repressor ZEB-1 to reduce expression of E-cadherin in colon cancer cells, increasing their invasive activity and reducing anoikis (23). CLDN1 mediates $\mathrm{TNF} \alpha$-induced gene expression and cell migration in human lung carcinoma cells (24). The CLDN1 expression was correlated with subtypes in breast $(25,26)$ and lung (27) cancer. Overexpression of CLDN1 in lung cancer cells inhibited cancer cell dissociation, and suppressed cancer cell migration, invasion and metastasis (14). CLDN1 expression can be regulated by many other transcriptional factors or signaling pathways. CLDN1 is involved in the $\beta$-catenin-T-cell factor/ lymphoid enhancing factor (TCF/LEF) signaling pathway (28). More recently, the expression of CLDN1 was found to be transcriptionally regulated by Cdx1, -2 and GATA4 (29). CLDN1 was regulated by Smad4 in a TGF $\beta$ signaling independent manner (30). Cdx2 can cooperate with Wnt pathway to regulate CLDN1 expression in colon cancer cells (29). The CLDN1 mRNA stability can also be regulated HDACdependently $(31,32)$.

To our knowledge, the immuno-profiles of CLDN1 and its association with $\beta$-catenin have not previously been reported in gastric cancer. Herein, we examined CLDN1 expression and correlations with clinicopathological characteristics, as well as its association with $\beta$-catenin in gastric cancer.

\section{Materials and methods}

Immunohistochemistry. Gastric cancer tissues, confirmed by pathological diagnosis, were obtained from 173 patients who underwent radical resection for gastric cancer at the Department of Surgery, Ruijin Hospital, Shanghai, China. The corresponding non-tumor gastric tissue was obtained at least $6 \mathrm{~cm}$ from the tumor. All tissue samples were formalin-fixed and paraffin-embedded. TNM staging was classified based on the criteria of American Joint Committee on Cancer (AJCC, 7th edition) for gastric cancer. The study was approved by the Shanghai Jiaotong University Medical School institutional review board.

Immunohistochemistry staining was performed by using a highly sensitive streptavidin-biotin-peroxidase detection system with gastric cancer tissue microarrays. Rabbit monoclonal anti-CLDN1 (working dilution 1:100) was purchased from Zymed (Invitrogen, USA) and rabbit anti- $\beta$-catenin (working dilution 1:100) was purchased from Cell Signaling (Danvers, MA, USA). Immunolabeling was conducted using Dako Envision + Rabbit Polymer (catalog no. K4003) from Dako (Carpinteria, CA, USA). The slides were counterstained with hematoxylin and coverslipped.

Immunohistochemistry scoring. The histology of the samples was examined by two histopathologists independently without knowing the clinicopathologic information. We scored the slides as previously described (33). The percentage of positive tumor cells was assigned to 5 categories: $\leq 5 \%$ (0); 5-25\% (1); $25-50 \%$ (2); $50-75 \%$ (3); and $\geq 75 \%$ (4). $\leq 5 \%$ positive cells were used as the cutoff to define negative tumors. The intensity of immunostaining was scored as: weak (1); moderate (2); and strong (3). The percentage of positivity of tumor cells and staining intensity were multiplied to produce a weighted score for each tumor specimen. The intensity scores were grouped as low (including scores 0 to +4 ) and high (including scores +6 to +12 ).

Cell culture. Human gastric cancer cell lines SGC-7901, MKN-28, MKN-45, BGC-823 and immortalized human gastric epithelial cell line GES-1 were obtained from Shanghai Institute of Cell Biology, Chinese Academy of Sciences. KATOIII, SNU-1, NCI-N87, HS-746T and AGS were obtained from American Type Culture Collection. The cells were grown in RPMI-1640 medium containing 10\% fetal bovine serum (FBS), penicillin and streptomycin (Gibco BRL, Gaithersburgh, MD, USA).

Western blotting. Whole cell lysates were harvested using RIPA cell lysis buffer supplemented with a protease inhibitor cocktail (Sigma-Aldrich, USA). Protein $(50 \mu \mathrm{g})$ was separated by SDS polyacrylamide gel electrophoresis and blotted onto $0.22-\mu \mathrm{m}$ polyvinylidene difluoride membranes (Millipore, MA, USA). Antibodies against CLDN1 (Zymed, USA) were used at 1:250 dilution. Antibodies against GAPDH (Sigma, St. Louis, MO, USA) were used at a 1:5,000 dilution. The signals were visualized using Li-COR Odyssey-Sa model 9260 (Li-COR Corp., USA) and images were taken and managed using Odyssey Sa Infrared Image System (Li-COR Corp.). Relative density of CLDN1 or $\beta$-catenin was measured by the following equation: Relative density $=$ density of CLDN1 or $\beta$-catenin band/density of GAPDH.

$\beta$-catenin knockdown and overexpression. After examining the expression of CLDN1 and $\beta$-catenin in nine gastric cancer cell lines (AGS, SNU-1, SGC-7901, MKN45, MKN28, KATO-III, HS-746T, BGC823 and NCI-N87) and one immortalized normal gastric epithelial cell line GES-1, we selected HS-746T as a model for $\beta$-catenin knockdown and overexpression assays to further investigate the relationship between $\beta$-catenin and CLDN1 because this cell line showed both membrane and nuclei expression of $\beta$-catenin. Short hairpin RNA (shRNA) lentiviral transduction particles for the $\beta$-catenin knockdown experiment was purchased from Genepharma (Shanghai, China). One $\beta$-catenin specific shRNA construct (shRNA sequence targeting $\beta$-catenin: GTGCTATCTGTCTGCTCTA) and one 'non-target' construct were transduced into HS-746T cells. The non-target construct contained an shRNA sequence (ACTACCGTTGTTATAGGTG) without targeting any known human gene and served as a scrambled negative control. After stable transfection into gastric cancer cell line HS-746T, cell clones of $\beta$-catenin knockdown (HS-746T/sh) and negative control (HS-746T/NC) were selected with puromycin (Sigma-Aldrich) at $10 \mu \mathrm{g} / \mathrm{ml}$ and screened by western blot analysis and immunofluorescence detection. To detect whether ectopic overexpression of $\beta$-catenin could restore the CLDN1 expression, the construct of LV5-EF1a-GFP/ $\beta$-catenin lentiviral transduction particles for ectopic overexpression of $\beta$-catenin was also purchased from Genepharma and transfected into HS-746T/sh cells. Stable transfected cell clone of HS-746T/sh/ $\beta$-catenin was selected with puromycin and screened by western blot analysis and immunofluorescence detection. 
Table I. The results of CLDN1 and $\beta$-catenin expression in immunohistochemical staining.

\begin{tabular}{|c|c|c|c|c|c|c|}
\hline & \multicolumn{2}{|c|}{ CLDN1 } & \multirow[b]{2}{*}{ P-value } & \multicolumn{2}{|c|}{$\beta$-catenin } & \multirow[b]{2}{*}{ P-value } \\
\hline & - & + & & - & + & \\
\hline Normal tissues & 91 & 82 & 0.002 & 86 & 87 & 0.040 \\
\hline Tumor tissues & 63 & 110 & & 67 & 106 & \\
\hline
\end{tabular}

Immunofluorescence staining. Cells were fixed with $4 \%$ formaldehyde and then permeabilized with PBS containing $0.2 \%$ Triton X-100. Slides were blocked by $5 \%$ BSA and incubated with a primary antibody at room temperature for $1 \mathrm{~h}$ followed by TRITC-labeled goat anti-rabbit IgG (Sigma) for additional $1 \mathrm{~h}$. Nucleus were counterstained using DAPI (Molecular Probes). Slides were washed by PBS, mounted and observed under a microscope. Immunofluorescence staining was visualized using Olympus BX50 microscope (Olympus Opticol Co., Japan), images were taken using Nikon Digital Sight DS-U2 (Nikon, Japan), and NIS elements F3.0 software was used (Nikon).

Statistical analysis. For IHC staining, the differences in clinicopathologic features between the different groups were determined using Pearson's $\chi^{2}$ test. $\mathrm{P}<0.05$ was considered to be statistically significant. The survival curves of each group were estimated by Kaplan-Meier survival analyses, and the curves were compared using log-rank tests. Statistical Package for the Social Sciences version 13.0 (SPSS, Inc., Chicago, IL, USA) was used for all statistical analyses.

\section{Results}

CLDN1 and $\beta$-catenin are overexpressed in gastric cancer tissues. Immunohistochemistry revealed that CLDN1 positive staining was localized in the membrane of tumor cells in gastric cancer. To investigate the correlations between CLDN1 and $\beta$-catenin, we performed IHC staining using the same cohort of tumor and non-tumor gastric specimens for $\beta$-catenin staining. In 173 gastric cancer tissues, $36.4 \%$ (63 of 173) of cases were CLDN1-negative (including no staining and cells stained $\leq 5 \%$ and staining intensity scored 1), while the remaining $63.6 \%$ (110 of 173) showed variable levels of CLDN1 expression, with a medium-score at 8.5 . Likewise, $38.7 \%$ (67 of 173) of cases were $\beta$-cateninnegative, while the remaining $61.3 \%$ (106 of 173) showed variable levels of $\beta$-catenin expression. In contrast, CLDN1 and $\beta$-catenin did not express, or were weakly expressed in normal gastric epithelial cells. As shown in Table I, CLDN1 was expressed in $47.4 \%$ of non-tumor tissues and $\beta$-catenin was expressed in $50.3 \%$ of non-tumor tissues. In summary, CLDN1 $(\mathrm{P}=0.002)$ and $\beta$-catenin $(\mathrm{P}=0.040)$ were highly expressed in gastric cancer tissues.

Characteristics of the expression profile of CLDN1 in gastric cancer tissues. We then investigated the clinicopathologic features of CLDN1 protein in human gastric cancer tissues. As shown in Table II, CLDN1 level decreased from well-moderately differentiated to poorly differentiated gastric cancer tissues $(\mathrm{P}=0.002)$. CLDN1 levels was higher in intestinal-type gastric cancer than that in diffuse-type gastric cancer $(\mathrm{P}=0.001)$. Higher $\mathrm{CLDN} 1$ expression levels were correlated with lymph node metastasis $(\mathrm{P}=0.042)$ and advanced TNM stage (stage III and IV) $(\mathrm{P}=0.030)$. CLDN1 expression was not significantly affected by gender $(\mathrm{P}=0.053)$, age $(\mathrm{P}=0.256)$, tumor size $(\mathrm{P}=0.966)$, $\mathrm{T}$ stage $(\mathrm{P}=0.386)$ and $M$ stage $(\mathrm{P}=0.936)$. These data suggested that CLDN1 was highly expressed in gastric cancer tissues with advanced clinicopathological features.

Characteristics of the expression profile of $\beta$-catenin in gastric cancer tissues. We next investigated the clinicopathologic features of $\beta$-catenin protein in human gastric cancer tissues. As shown in Table II, $\beta$-catenin level was correlated with older age $(\mathrm{P}=0.008)$, differentiation $(\mathrm{P}<0.0001)$ and intestinal-type gastric cancer $(\mathrm{P}<0.0001)$. Higher $\beta$-catenin expression levels were correlated with lymph node metastasis $(\mathrm{P}=0.016)$ and advanced TNM stage (stage III and IV) $(\mathrm{P}=0.043) . \beta$-catenin expression was not significantly affected by gender $(\mathrm{P}=0.549)$, tumor size $(\mathrm{P}=0.116)$, $\mathrm{T}$ stage $(\mathrm{P}=0.326)$ and $\mathrm{M}$ stage $(\mathrm{P}=0.928)$. These data suggested that $\beta$-catenin was highly expressed in gastric cancer tissues with advanced clinicopathological features.

Correlation between CLDN1 with $\beta$-catenin levels in gastric cancer tissues. Previous studies have shown that CLDN1 can be regulated by $\beta$-catenin (28). To investigate the association of CLDN1 and $\beta$-catenin, we performed IHC staining using the same cohort of specimens as we used for CLDN1 staining. As expected, we observed positive correlation between the expression levels of CLDN1 and $\beta$-catenin in gastric cancer analyzed by IHC staining. As presented in Fig. 1B-D, one specimen with high CLDN1 level also showed high $\beta$-catenin level, while another specimen with very weak CLDN1 expression also showed nearly negative $\beta$-catenin level. The correlation of CLDN1 and $\beta$-catenin was further analyzed using the Person correlation. As shown in Fig. 2, y-axis presents the IHC scores of $\beta$-catenin and $x$-axis presents the CLDN1 IHC scores using the same cohort of gastric cancer specimens. Linear trend line showed CLDN1 levels were positively correlated to $\beta$-catenin levels. Person correlation analysis showed correlation coefficient of 0.637 ( $\mathrm{P}<0.0001)$. These data suggested that CLDN1 expression levels were positively correlated with $\beta$-catenin levels.

Interestingly, we also established that CLDN1 was correlated with the subcellular location of $\beta$-catenin, which might suggest the activation of $\beta$-catenin related signaling pathway. Among these 106 cases of $\beta$-catenin positive staining, 72 cases displayed membrane staining of $\beta$-catenin, while 34 cases showed nuclear $\beta$-catenin. As shown in Fig. $1 \mathrm{C}$, strong CLDN1 expression was observed in an gastric cancer specimen (left), and nuclei localized $\beta$-catenin was also observed in the same case (right).

CLDN1 and $\beta$-catenin levels predict survival in gastric cancer patients. To investigate the prognostic significance of CLDN1 and $\beta$-catenin in gastric cancer, we analyzed the correlation 
Table II. Clinicopathological associations of CLDN1 and $\beta$-catenin expression in gastric cancer.

\begin{tabular}{|c|c|c|c|c|c|c|}
\hline & \multicolumn{2}{|c|}{ CLDN1 } & \multirow[b]{2}{*}{ P-value } & \multicolumn{2}{|c|}{$\beta$-catenin } & \multirow[b]{2}{*}{ P-value } \\
\hline & - & + & & - & + & \\
\hline \multicolumn{7}{|l|}{ Gender } \\
\hline Male & 50 & 72 & 0.053 & 49 & 73 & 0.549 \\
\hline Female & 13 & 38 & & 18 & 33 & \\
\hline \multicolumn{7}{|l|}{ Age } \\
\hline$\leq 65$ & 36 & 53 & 0.256 & 43 & 46 & 0.008 \\
\hline$>65$ & 27 & 57 & & 24 & 60 & \\
\hline \multicolumn{7}{|l|}{ Tumor size $(\mathrm{cm})$} \\
\hline$\leq 5$ & 34 & 59 & 0.966 & 31 & 62 & 0.116 \\
\hline$>5$ & 29 & 51 & & 36 & 44 & \\
\hline \multicolumn{7}{|l|}{ Differentiation } \\
\hline Well + moderate & 22 & 65 & 0.002 & 19 & 68 & 0.000 \\
\hline Poor & 41 & 45 & & 48 & 38 & \\
\hline \multicolumn{7}{|l|}{ Lauren classification } \\
\hline Intestinal & 34 & 85 & 0.001 & 31 & 90 & 0.000 \\
\hline Diffuse & 29 & 25 & & 36 & 16 & \\
\hline \multicolumn{7}{|l|}{ T stage } \\
\hline $\mathrm{T} 1+\mathrm{T} 2$ & 13 & 17 & 0.386 & 14 & 16 & 0.326 \\
\hline $\mathrm{T} 3+\mathrm{T} 4$ & 50 & 93 & & 53 & 90 & \\
\hline \multicolumn{7}{|l|}{ TNM stage } \\
\hline $\mathrm{I}+\mathrm{II}$ & 28 & 31 & 0.030 & 29 & 30 & 0.043 \\
\hline $\mathrm{III}+\mathrm{IV}$ & 35 & 79 & & 38 & 76 & \\
\hline \multicolumn{7}{|c|}{ Lymph node metastasis } \\
\hline No & 20 & 20 & 0.042 & 22 & 18 & 0.016 \\
\hline $\mathrm{N} 1+\mathrm{N} 2+\mathrm{N} 3$ & 43 & 90 & & 45 & 88 & \\
\hline \multicolumn{7}{|l|}{ Distance metastasis } \\
\hline M0 & 59 & 102 & 0.936 & 63 & 98 & 0.928 \\
\hline M1 & 4 & 8 & & 4 & 8 & \\
\hline
\end{tabular}

of CLDN1 and $\beta$-catenin with survival of the patients using Kaplan-Meier analysis. Patients in the CLDN1-positive group showed shorter overall survival than those in CLDN1-negative group (medium survival: 44.1 vs. 51.5 months, $\mathrm{P}=0.045$, Fig. 3A, left). However, $\beta$-catenin-positive patients showed a trend of shorter overall survival (medium survival: 44.6 vs. 49.6 months, $\mathrm{P}=0.113$, Fig. $3 \mathrm{~A}$, right). CLDN1 and $\beta$-catenin levels were significantly associated with Lauren classification (Table II), therefore, we next stratified patients with intestinaland diffuse-type to investigate the prognostic value of CLDN1 and $\beta$-catenin. In intestinal-type patients, both CLDN1positive group (medium survival: 45.4 vs. 55.3 months, $\mathrm{P}=0.043$, Fig. 3B, left) and $\beta$-catenin-positive group (medium survival: 45.3 vs. 56.4 months, $\mathrm{P}=0.021$, Fig. $3 \mathrm{~B}$, right) were significantly correlated with shorter overall survival. However, in diffuse-type patients, no statistically significant difference was found (Fig. 3C, left and right). Our data suggested the expression of CLDN1 and $\beta$-catenin was significantly associated with shorter overall survival in intestinal-type gastric cancer patients.

$\beta$-catenin knockdown or ectopic overexpression regulates CLDN1 levels. As previous studies suggested that $\beta$-catenin could regulate CLDN1 expression, we then performed $\beta$-catenin knockdown using pre-designed shRNA to examine the CLDN1 level. We employed HS-746T as a model because this cell line has shown both membrane and nuclear location of $\beta$-catenin, which suggested a potential activation of $\beta$-catenin transcriptional activity. Immunoblotting and immunofluorescence staining was used to observe the expression level and subcellular location of $\beta$-catenin and CLDN1 in control-shRNA (HS-746T/NC) and $\beta$-catenin-shRNA (HS-746T/sh) transfected cells using anti-CLDN1 and anti$\beta$-catenin antibodies. $\beta$-catenin was barely observed after 

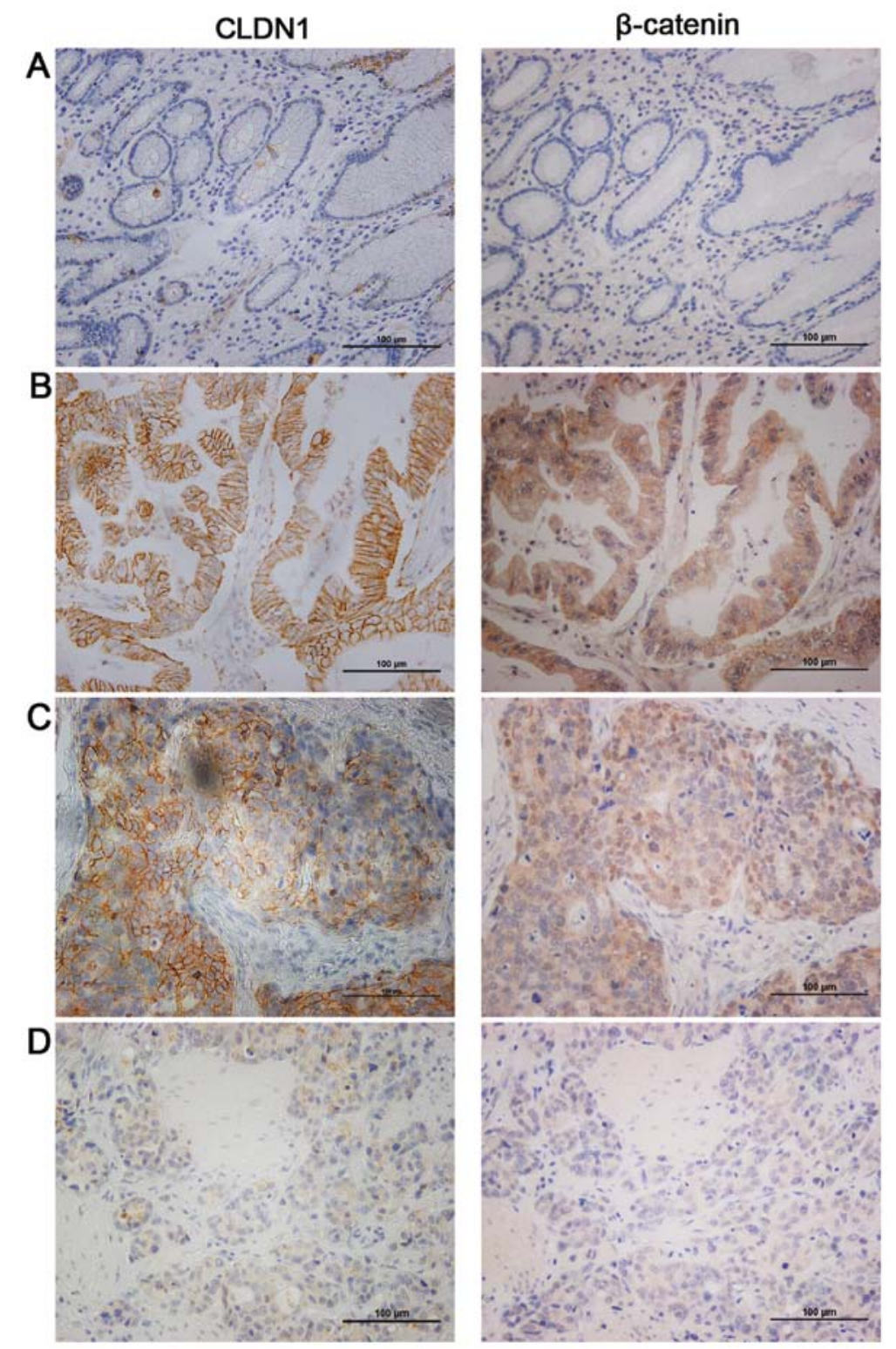

Figure 1. Expression of CLDN1 and $\beta$-catenin in gastric cancer and normal gastric mucosa was detected by IHC staining (original magnifications, $\mathrm{x} 200$ ). (A) Normal gastric mucosa showed negative staining. (B and C) Positive staining in gastric cancer. (D) Negative staining in gastric cancer.

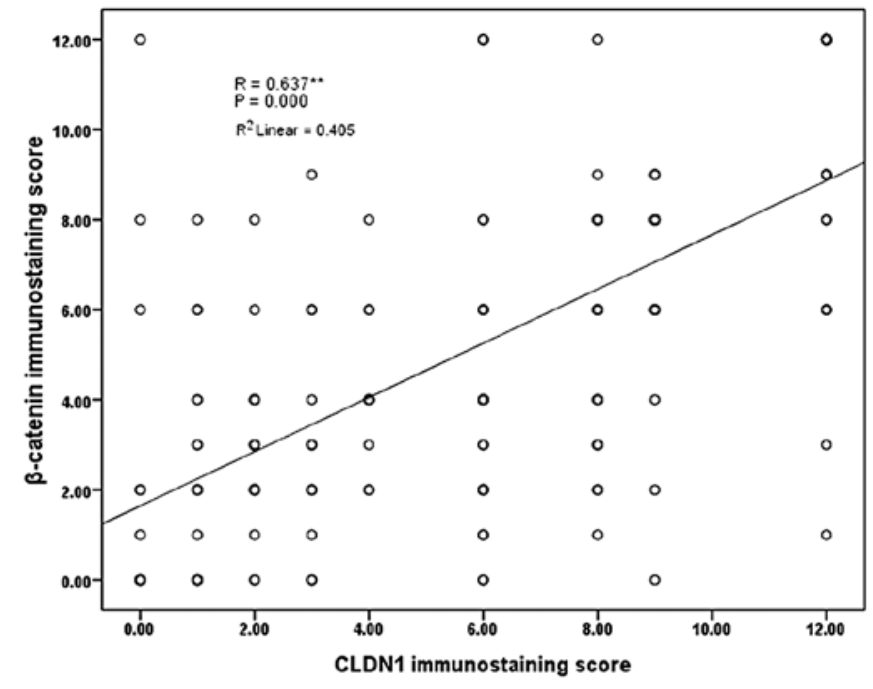

Figure 2. The correlation of CLDN1 and $\beta$-catenin IHC staining scores.
$\beta$-catenin-shRNA transfection (Figs. 4 and 5B), and the CLDN1 level was dramatically decreassed (Figs. 4 and 5E). We next ask whether ectopic overexpression of $\beta$-catenin could restore the CLDN1 expression. To do so, we transfected $\beta$-catenin full-length cDNA into HS-746T/sh cells. As shown in Figs. 4 and 5C and F, CLDN1 expression was significantly increased when HS-746T/sh cells were transfected with $\beta$-catenin construct. These data strongly suggested that CLDN1 level was regulated by $\beta$-catenin. Nuclei localized $\beta$-catenin showed moderate staining in HS-746T/NC (Fig. 5A) and strong staining in HS-746T/sh/ $\beta$-catenin (Fig. 5C) which might be an activation of transcriptional activity contributing to the regulation of CLDN1 expression.

\section{Discussion}

CLDN1 can positively and negatively regulate tumorigenesis in different human cancer types. $\beta$-catenin, a multifunc- 

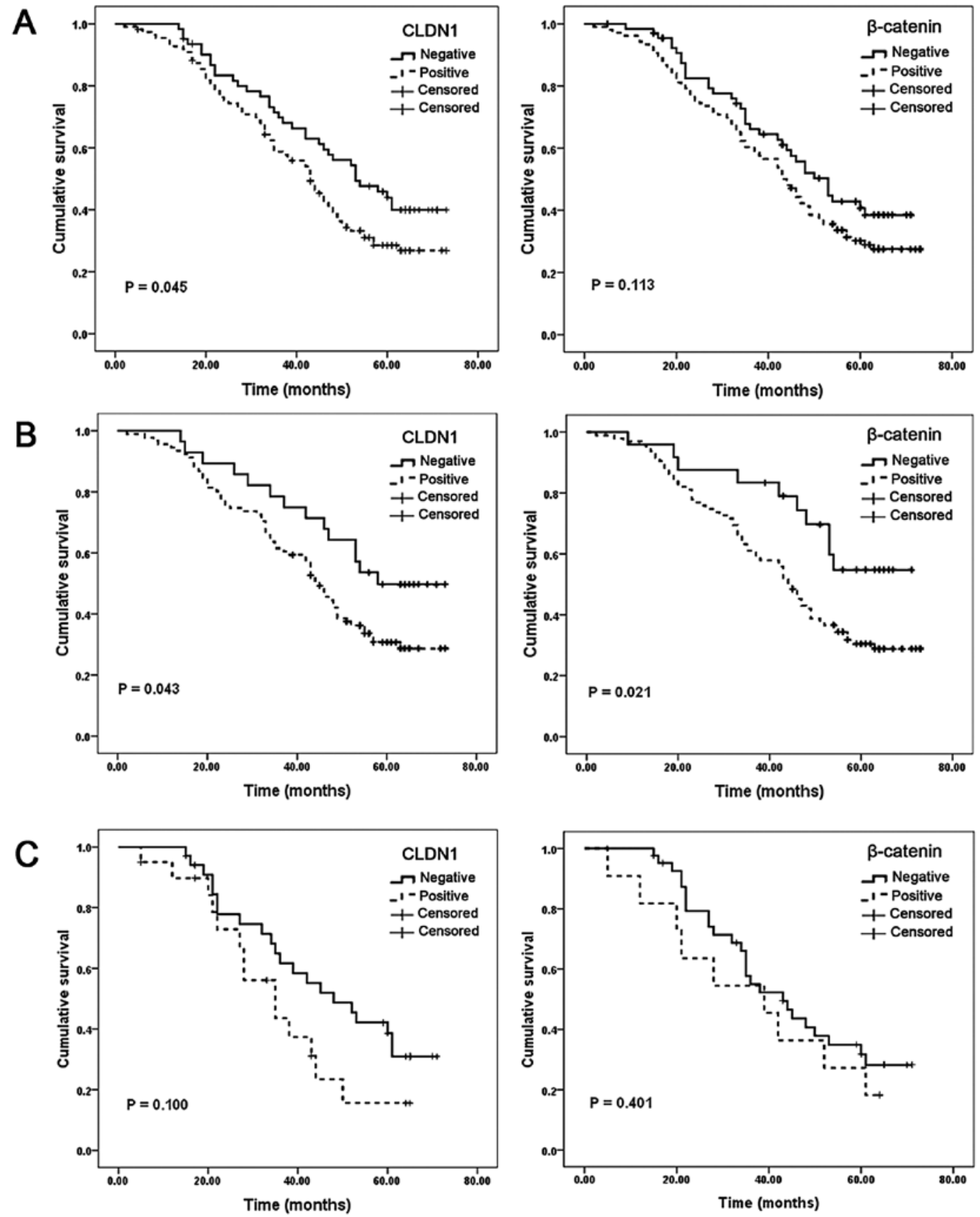

Figure 3. Kaplan-Meier curves of overall survival of gastric cancer patients, stratified by CLDN1 and $\beta$-catenin levels. (A) Survival distribution in total gastric cancer patients. (B) Survival distribution in intestinal-type gastric cancer patients. (C) Survival distribution in diffuse-type gastric cancer patients.

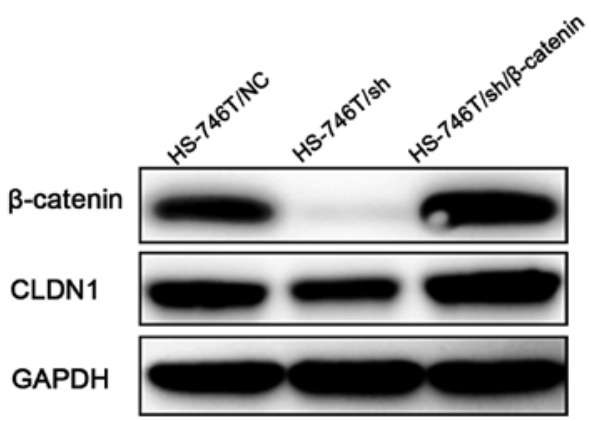

Figure 4. Immunoblotting of $\beta$-catenin and CLDN1 in the cell line HS-746T with knockdown and overexpression of $\beta$-catenin. $\beta$-catenin knockdown and ectopic overexpression decreased and increased CLDN1 levels, respectively. GAPDH was used as loading control. tional protein, is found to be critical in tumor development and progression. We showed CLDN1 and $\beta$-catenin levels were positively correlated. The expression of both CLDN1 and $\beta$-catenin were associated with tumor differentiation, Lauren classification, TNM stage and lymph node metastasis. Furthermore, CLDN1 and $\beta$-catenin were predictive factors for shorter overall survival in gastric patients. Finally, CLDN1 levels were regulated by $\beta$-catenin.

CLDN1 is a member of tight junction protein which generally plays a role in maintaining the integrity of the barrier function in normal epithelial cells (34). However, in tumor cells, CLDN1 was discovered to be either a promoter or an inhibitor. Because CLDN1-induced acquisition of the malignant EMT phenotype, 

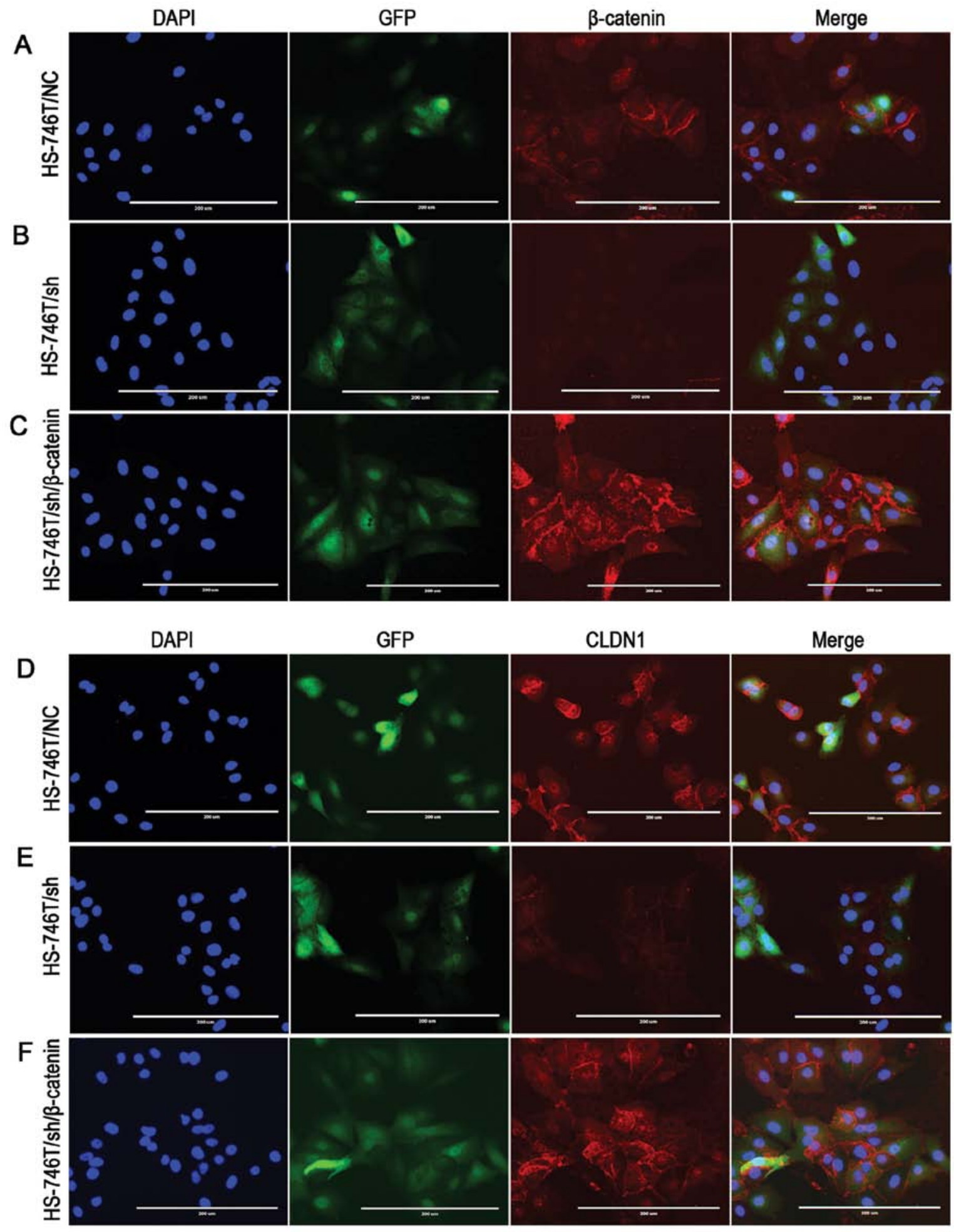

Figure 5. Immunofluorescence staining of $\beta$-catenin and CLDN1 in the cell line HS-746T with knockdown and overexpression of $\beta$-catenin. $\beta$-catenin (red) expression was significant decreased in HS-746T/sh (B) and higher expressed both on membrane and in nuclei in HS-746T/ $\beta$-catenin (C) as compared to negative control HS-746T/NC cells (A). CLDN1 level was dramatically decreased (E) and increased (F) as compared to negative control HS-746T/NC cells (D), following knockdown and overexpression of $\beta$-catenin, respectively.

it was exploited as a biomarker for metastasis in liver cancer $(21,35,36)$. Increased CLDN1 protein expression was related to metastasis capacity of colorectal cancer (16,30). Chang et al discovered that CLDN1 had tumor suppressive activity and was a direct transcriptional target of RUNX3 in gastric cancer cells (12). They also found that knockdown of claudin 1 increased the tumorigenicity of human gastric cancer cells (12). However, study also found that overexpression of other members of claudin (CLDN6, 7 and 9) in gastric cancer cells increased the invasiveness, migration and proliferation (37).

We showed that CLDN1 was highly expressed in gastric cancer tissues that were intestinal-type, differentiated, in 
advanced TNM stage and with lymph node metastasis, furthermore, CLDN1 showed its prognostic significance especially in intestinal-type of gastric cancer. Our result is consistent with earlier findings which showed loss of CLDN1 in diffuse-type of gastric cancer in patients from Finland and United States $(38,39)$. However, the prognostic significance of CLDN1 in our study was the first observation. This may be caused by the difference of patient nationality, because Chinese mainland patients were enrolled in our study. Our finding was thus supported by another Chinese group which showed that CLDN1 expression was correlated with differentiation, invasiveness and metastasis of gastric carcinoma (40).

Accumulation of $\beta$-catenin in cytoplasm and nuclei is frequently observed in a wide variety of tumors which reflects the stabilization and activation of $\beta$-catenin $(41,42)$. The subsequent activation of $\mathrm{Wnt} / \beta$-catenin signaling pathway is common in majority of gastric cancers (43). CLDN1 is a known target of $\beta$-catenin/TCF/LEF-dependent transcription regulation (28). Our findings that $\beta$-catenin levels were associated with differentiation, TNM stage, lymph node metastasis shared similar pattern with CLDN1. Both CLDN1 and $\beta$-catenin predicted shorter survival in intestinal-type gastric cancer patients. Protein levels of CLDN1 and $\beta$-catenin were positively correlated in gastric cancer. These findings strongly suggested a connection between these two markers. However, CLDN1 was a predictive factor for all patients suggesting it as a better prognostic factor compared with $\beta$-catenin. We also found that the CLDN1 level was regulated by $\beta$-catenin. CLDN1 levels decreased with knockdown of $\beta$-catenin, while CLDN1 level was elevated when $\beta$-catenin level was increased by overexpression. These findings were consistent with other studies and our present study which suggested a positively correlation of CLDN1 and $\beta$-catenin.

In conclusion, our study reveals CLDN1 as a negative prognostic factor which predicts shorter overall survival in gastric cancer patients. Clinicopathologic features of CLDN1 were similar to those of $\beta$-catenin in gastric cancer. CLDN1 levels in gastric cancer tissues were positively correlated with $\beta$-catenin levels. Furthermore, CLDN1 levels were regulated by $\beta$-catenin. However, CLDN1 severs as a better prognostic factor than $\beta$-catenin in gastric cancer patients.

\section{References}

1. Brenner H, Rothenbacher D and Arndt V: Epidemiology of stomach cancer. Methods Mol Biol 472: 467-477, 2009.

2. Du C, Zhou Y, Cai H, Zhao G, Fu H and Shi YQ: Poor prognostic factors in patients with stage I gastric cancer according to the seventh edition TNM classification: a comparative analysis of three subgroups. J Surg Oncol 105: 323-328, 2012.

3. Lazar D, Taban S, Sporea I, et al: Gastric cancer: correlation between clinicopathological factors and survival of patients (III). Rom J Morphol Embryol 50: 369-379, 2009.

4. Lazar D, Taban S, Sporea I, et al: Gastric cancer: correlation between clinicopathological factors and survival of patients. II. Rom J Morphol Embryol 50: 185-194, 2009.

5. Tsukita $\mathrm{S}$ and Furuse M: Claudin-based barrier in simple and stratified cellular sheets. Curr Opin Cell Biol 14: 531-536, 2002.

6. Tobioka H, Isomura H, Kokai Y, Tokunaga Y, Yamaguchi J and Sawada N: Occludin expression decreases with the progression of human endometrial carcinoma. Hum Pathol 35: 159-164, 2004.

7. Pope JL, Bhat AA, Sharma A, et al: Claudin-1 regulates intestinal epithelial homeostasis through the modulation of Notch-signalling. Gut: Jun 13, 2013 (Epub ahead of print).
8. Noda S, Tanabe S and Suzuki T: Naringenin enhances intestinal barrier function through the expression and cytoskeletal association of tight junction proteins in Caco-2 cells. Mol Nutr Food Res 57: 2019-2028, 2013

9. Iraha A, Chinen H, Hokama A, et al: Fucoidan enhances intestinal barrier function by upregulating the expression of claudin-1. World J Gastroenterol 19: 5500-5507, 2013.

10. Myal Y, Leygue E and Blanchard AA: Claudin 1 in breast tumorigenesis: revelation of a possible novel 'claudin high' subset of breast cancers. J Biomed Biotechnol 2010: 956897, 2010.

11. Blanchard AA, Ma X, Dueck KJ, et al: Claudin 1 expression in basal-like breast cancer is related to patient age. BMC Cancer 13: 268, 2013.

12. Chang TL, Ito K, Ko TK, et al: Claudin-1 has tumor suppressive activity and is a direct target of RUNX3 in gastric epithelial cells. Gastroenterology 138: 255-265, 2010.

13. Qin W, Ren Q, Liu T, Huang Y and Wang J: MicroRNA-155 is a novel suppressor of ovarian cancer-initiating cells that targets CLDN1. FEBS Lett 587: 1434-1439, 2013.

14. Chao YC, Pan SH, Yang SC, et al: Claudin-1 is a metastasis suppressor and correlates with clinical outcome in lung adenocarcinoma. Am J Respir Crit Care Med 179: 123-133, 2009.

15. Dhawan P, Singh AB, Deane NG, et al: Claudin-1 regulates cellular transformation and metastatic behavior in colon cancer. J Clin Invest 115: 1765-1776, 2005.

16. Kinugasa T, Akagi Y, Ochi T, et al: Increased claudin-1 protein expression in hepatic metastatic lesions of colorectal cancer. Anticancer Res 32: 2309-2314, 2012.

17. Ersoz S, Mungan S, Cobanoglu U, Turgutalp H and Ozoran Y: Prognostic importance of Claudin-1 and Claudin-4 expression in colon carcinomas. Pathol Res Pract 207: 285-289, 2011.

18. Morohashi S, Kusumi T, Sato F, et al: Decreased expression of claudin-1 correlates with recurrence status in breast cancer. Int J Mol Med 20: 139-143, 2007.

19. Zhang Z, Wang A, Sun B, Zhan Z, Chen K and Wang C: Expression of CLDN1 and CLDN10 in lung adenocarcinoma in situ and invasive lepidic predominant adenocarcinoma. J Cardiothorac Surg 8: 95, 2013.

20. Matsuda Y, Semba S, Ueda J, et al: Gastric and intestinal claudin expression at the invasive front of gastric carcinoma. Cancer Sci 98: 1014-1019, 2007.

21. Suh Y, Yoon CH, Kim RK, et al: Claudin-1 induces epithelialmesenchymal transition through activation of the c-Abl-ERK signaling pathway in human liver cells. Oncogene 32: 4873-4882, 2013.

22. Singh AB, Sharma A and Dhawan P: Claudin-1 expression confers resistance to anoikis in colon cancer cells in a Src-dependent manner. Carcinogenesis 33: 2538-2547, 2012.

23. Singh AB, Sharma A, Smith JJ, et al: Claudin-1 up-regulates the repressor ZEB-1 to inhibit E-cadherin expression in colon cancer cells. Gastroenterology 141: 2140-2153, 2011.

24. Shiozaki A, Bai XH, Shen-Tu G, et al: Claudin 1 mediates TNFalpha-induced gene expression and cell migration in human lung carcinoma cells. PLoS One 7: e38049, 2012.

25. Di Cello F, Cope L, Li H, et al: Methylation of the claudin 1 promoter is associated with loss of expression in estrogen receptor positive breast cancer. PLoS One 8: e68630, 2013.

26. Blanchard AA, Skliris GP, Watson PH, et al: Claudins 1, 3, and 4 protein expression in ER negative breast cancer correlates with markers of the basal phenotype. Virchows Arch 454: 647-656, 2009.

27. Moldvay J, Jackel M, Paska C, Soltesz I, Schaff Z and Kiss A: Distinct claudin expression profile in histologic subtypes of lung cancer. Lung Cancer 57: 159-167, 2007.

28. Miwa N, Furuse M, Tsukita S, Niikawa N, Nakamura Y and Furukawa Y: Involvement of claudin-1 in the beta-catenin/ Tcf signaling pathway and its frequent upregulation in human colorectal cancers. Oncol Res 12: 469-476, 2001.

29. Bhat AA, Sharma A, Pope J, et al: Caudal homeobox protein Cdx-2 cooperates with Wnt pathway to regulate claudin-1 expression in colon cancer cells. PLoS One 7: e37174, 2012.

30. Shiou SR, Singh AB, Moorthy K, et al: Smad4 regulates claudin-1 expression in a transforming growth factor-beta-independent manner in colon cancer cells. Cancer Res 67: 1571-1579, 2007.

31. Sharma A, Bhat AA, Krishnan M, Singh AB and Dhawan P: Trichostatin-A modulates claudin-1 mRNA stability through the modulation of $\mathrm{Hu}$ antigen $\mathrm{R}$ and tristetraprolin in colon cancer cells. Carcinogenesis 34: 2610-2621, 2013. 
32. Krishnan M, Singh AB, Smith JJ, et al: HDAC inhibitors regulate claudin-1 expression in colon cancer cells through modulation of mRNA stability. Oncogene 29: 305-312, 2010.

33. Sinicrope FA, Ruan SB, Cleary KR, Stephens LC, Lee JJ and Levin B: bcl-2 and p53 oncoprotein expression during colorecta tumorigenesis. Cancer Res 55: 237-241, 1995.

34. Mrsny RJ, Brown GT, Gerner-Smidt K, et al: A key claudin extracellular loop domain is critical for epithelial barrier integrity. Am J Pathol 172: 905-915, 2008.

35. Stebbing J, Filipovic A and Giamas G: Claudin-1 as a promoter of EMT in hepatocellular carcinoma. Oncogene 32: 4871-4872, 2013.

36. Yoon CH, Kim MJ, Park MJ, et al: Claudin-1 acts through c-Ablprotein kinase Cdelta (PKCdelta) signaling and has a causal role in the acquisition of invasive capacity in human liver cells. J Biol Chem 285: 226-233, 2010.

37. Zavala-Zendejas VE, Torres-Martinez AC, Salas-Morales B Fortoul TI, Montano LF and Rendon-Huerta EP: Claudin-6, 7 , or 9 overexpression in the human gastric adenocarcinoma cell line AGS increases its invasiveness, migration, and proliferation rate. Cancer Invest 29: 1-11, 2011.
38. Soini Y, Tommola S, Helin $\mathrm{H}$ and Martikainen P: Claudins 1, 3, 4 and 5 in gastric carcinoma, loss of claudin expression associates with the diffuse subtype. Virchows Arch 448: 52-58, 2006.

39. Resnick MB, Gavilanez M, Newton E, et al: Claudin expression in gastric adenocarcinomas: a tissue microarray study with prognostic correlation. Hum Pathol 36: 886-892, 2005.

40. Wu YL, Zhang S, Wang GR and Chen YP: Expression transformation of claudin-1 in the process of gastric adenocarcinoma invasion. World J Gastroenterol 14: 4943-4948, 2008.

41. Demir R, Dimmler A, Naschberger E, et al: Malignant progression of invasive tumour cells seen in hypoxia present an accumulation of beta-catenin in the nucleus at the tumour front. Exp Mol Pathol 87: 109-116, 2009.

42. Li YP, Wu CC, Chen WT, Huang YC and Chai CY: The expression and significance of WWOX and beta-catenin in hepatocellular carcinoma. APMIS 121: 120-126, 2013.

43. Ooi $\mathrm{CH}$, Ivanova $\mathrm{T}$, Wu J, et al: Oncogenic pathway combinations predict clinical prognosis in gastric cancer. PLoS Genet 5: e1000676, 2009. 